\title{
Comparison of TNM staging systems for nasopharyngeal carcinoma, and proposal of a new staging system
}

\author{
P-Y OuYang ${ }^{1}, \mathrm{Z} \mathrm{Su}^{1}, \mathrm{X}-\mathrm{H} \mathrm{Ma}{ }^{2}, \mathrm{Y}-\mathrm{P}$ Mao ${ }^{1}, \mathrm{M}-\mathrm{Z} \mathrm{Liu}^{*, 1,3}$ and F-Y Xie ${ }^{*, 1,3}$
}

${ }^{1}$ Department of Radiation Oncology, Sun Yat-sen University Cancer Center, State Key Laboratory of Oncology in South China, Collaborative Innovation Center for Cancer Medicine, No. 651 Dongfeng Road East, Guangzhou 510060, Guangdong, China and ${ }^{2}$ Department of Medical Statistics and Epidemiology, School of Public Health, Sun Yat-sen University, Guangzhou, Guangdong, China

Background: There are few systematic evaluations regarding the sixth and seventh editions of the UICC/AJCC TNM Staging System (TNM6th, TNM7th) and Chinese 2008 Staging System (TNMc2008) for nasopharyngeal carcinoma (NPC).

Methods: We classified 2333 patients into intensity-modulated radiotherapy (IMRT) cohort $(n=941)$ and conventional radiotherapy (CRT) cohort $(n=1392)$. Tumour staging defined by TNM6th, TNM7th and TNMc2008 was compared based on Akaike information criterion (AIC) and Harrell's concordance index (c-index).

Results: For T-classification, TNM6th $(\mathrm{AIC}=2585.367$; $\mathrm{c}$-index $=0.6390385)$ had superior prognostic value to TNM7th $(A I C=2593.242 ; \quad c$-index $=0.6226889)$ and TNMc2008 (AIC $=2593.998 ; \quad c$-index $=0.6237146)$ in the IMRT cohort, whereas TNMc2008 was superior ( $\mathrm{AIC}=5999.054$; $c$-index $=0.623547$ ) in the CRT cohort. For N-classification, TNMc2008 had the highest prognostic value in both cohorts $(\mathrm{AIC}=2577.726$, $c$-index $=0.6297874$; $\mathrm{AIC}=5956.339$, $\mathrm{c}$-index $=0.6533576)$. Similar results were obtained when patients were stratified by chemotherapy types, age and gender. Using staging models in the IMRT cohort, we failed to identify better stage migrations than TNM6th T-classification and TNMc2008 N-classification. We therefore proposed to combine these categories; resultantly, stage groups of the proposed staging system showed superior prognostic value over TNM6th, TNM7th and TNMc2008.

Conclusion: TNM6th T-classification and TNMc2008 N-classification have superior prognostic value in the IMRT era. By combining them with slight modifications, TNM criteria can be unified and its prognostic value be improved.

\begin{abstract}
Nasopharyngeal carcinoma (NPC) is a non-lymphomatous, squamous-cell carcinoma that occurs in the epithelial lining of the nasopharynx. It has a distinct epidemiology, aetiology (Chang and Adami, 2006) and clinical manifestation (Wei and Sham, 2005) compared with other cancers, including other types of head and neck cancers. The highest rates of incidence occur in Southeast Asia, especially in Southern China, where the incidence of NPC can be as high as 20 to 30 per 100000 (Cao et al, 2011). In contrast, $\mathrm{NPC}$ is relatively rare in Europe and the United States, where the incidence is only 0.5 to 2 per 100000 (Ferlay et al, 2004).
\end{abstract}

An accurate staging system is critical for defining prognosis, determining appropriate treatment and evaluating treatment outcomes. The introduction of the sixth edition of the TNM staging system (TNM6th) for NPC (Greene et al, 2002), jointly adopted by the International Union against Cancer (UICC) and American Joint Committee for Cancer (AJCC), was an important landmark. Subsequent retrospective studies showed that nasal cavity/oropharynx involvement without parapharyngeal extension (T2a by TNM6th) had a similar, favourable prognosis to T1 (Lee et al, 2004; Liu et al, 2008; Mao et al, 2009); anatomic masticator

\footnotetext{
*Correspondence: Professor F-Y Xie or Professor M-Z Liu; E-mail: xiefy@sysucc.org.cn or liumzh@sysucc.org.cn

${ }^{3}$ These authors contributed equally to this work.
}

Received 5 July 2013; revised 25 September 2013; accepted 1 October 2013; published online 22 October 2013 
space involvement including the medial and lateral pterygoid muscles had a similar prognosis to T4 (Tang et al, 2010); and retropharyngeal lymph node (RP-LN) metastasis, regardless of laterality, had a poorer prognosis than node-negative disease (Tang et al, 2008; Tham et al, 2009). Therefore, the recent seventh edition of the UICC/AJCC (TNM7th) (Edge et al, 2009, 2010) revised the criteria based on these findings. However, reverse evidence suggested that T2a patients should not be in the same prognostic group as T1 patients (Low et al, 2004); studies (Chua et al, 1997; $\mathrm{Ng}$ et al, 2007; Sun et al, 2013) revealed that RP-LN metastasis did not influence overall survival (OS) and distant metastasis-free survival (DMFS). In addition, with the enhanced locoregional control of intensity-modulated radiotherapy (IMRT), it needs a full reappraisal to see whether the prognosis of the medial and lateral pterygoid muscles involvement remains to be similar to T4. Finally, as NPC is especially prevalent in Southern China (Cao et al, 2011), the Chinese 2008 Staging System for NPC (TNMc2008) (CCSNPC, 2013) was released by the Chinese Committee for Staging of Nasopharyngeal Carcinoma, based on the Chinese 1992 Staging System.

Although TNM7th is now internationally recommended, TNMc2008 is widely used in the most endemic area - Mainland China. This discrepancy is because scientific evidence has not satisfactorily justified the use of these criteria in cancer staging. It greatly influences treatment assessment, and comparisons and clinical cooperation between different centres. Identification of the most useful staging criteria for therapeutic decision making is warranted. Few carefully designed studies have formally compared the two editions of UICC/AJCC staging system and the Chinese 2008 staging system to determine which is most useful for therapeutic decision making. Therefore, we performed this study to comprehensively evaluate and directly compare the three NPC staging systems - TNM6th, TNM7th and TNMc2008. In addition, considering the discrepancies between TNM6th, TNM7th and TNMc2008 and proposals from studies (Hu et al, 2010; Tang et al, 2010; Lee et al, 2012; Chen et al, 2012a,b; Li et al, 2013; Sun et al, 2013), we constructed staging models, compared them with the existing staging systems and ultimately proposed and confirmed some modifications for NPC staging system.

\section{MATERIALS AND METHODS}

Patients. The study was reviewed and approved by the Human Ethics Approval Committee at Sun Yat-sen University Cancer Center. We retrospectively reviewed the medical records of 2333 patients with newly diagnosed, biopsy-proven, non-metastatic NPC hospitalised at our centre. We classified them into three cohorts. The first one - IMRT cohort - involved 941 patients (714 men, 227 women; median age, 46 years; range, 13-84 years; histological type: I, 1; II, 35; and III, 905) treated with IMRT between January 2003 and December 2009. The second one - CRT cohort - involved 1392 patients (1054 men, 338 women; median age, 45 years; range, $12-80$ years; type: I, 5; II, 70; and III, 1317) treated with conventional radiotherapy (CRT) between January 2005 and December 2006. To validate the results of the IMRT and CRT cohorts, we enrolled 1673 patients with NPC treated between January 2005 and December 2006, irrespective of radiation techniques, as the third cohort (1269 men, 404 women; median age, 45 years; range, $12-80$ years; type: I, 6; II, 91; and III, 1576; IMRT, 281 (16.8\%), CRT, $1392(83.2 \%))$.

All included patients had complete pretreatment evaluation including patient history, physical examination, haematology and biochemistry profiles, fiberoptic nasopharyngoscopy with biopsy, magnetic resonance imaging (MRI) of the nasopharynx and neck, chest radiography, abdominal sonography and Technetium-99m- methylene diphosphonate (Tc-99-MDP) whole-body bone scan. In addition, a total of 199 (8.5\% of 2333) patients underwent $18 \mathrm{~F}$ fluorodeoxyglucose positron emission tomography and computed tomography (PET/CT) - 132 (14.0\% of 941$), 67$ (4.8\% of 1392) and $141(8.4 \%$ of 1673$)$ patients in the IMRT, CRT and the third cohort, respectively. Two radiologists independently reviewed all the images based on the MRI diagnosis criteria (see Supplementary Information) (King, 2010) and restaged all the patients according to the criteria of the three staging systems (Table 1). Any disagreements were resolved by consensus.

All patients were treated by definitive IMRT or CRT with or without chemotherapy; the radiation techniques and chemotherapy regimens have been described previously (Ma et al, 2007; Liang et al, 2009; Chen et al, 2012b). Considering the heterogeneous chemotherapy regimens, subgroup analysis by chemotherapy (induction chemotherapy (IC); concomitant chemotherapy (CC); IC plus CC; and CC plus adjuvant chemotherapy) was conducted in each cohort. In addition, stratified analysis by age and gender was also performed.

Patients were examined every 3-6 months during the first 3 years, with follow-up examinations every 6-12 months thereafter or until death. Patients without recent examination tests in the medical records were followed up by telephone call till June of this year. Within the median follow-up duration (from the first day of therapy) of 57 months (range, 3-124 months), 74 months (range, 2-102 months) and 74 months (range, 2-102 months) for the IMRT, CRT and the third cohort, 102 out of 941 (10.8\%), 143 out of $1392(10.3 \%)$ and 174 out of $1673(10.4 \%)$ patients were lost to follow-up, with 200, 435 and 510 cases of treatment failure (locoregional relapse, distant metastasis or death from any cause, whichever was first; the diagnosing criteria were available in Supplementary Information), respectively.

Statistical analysis. Statistical analyses were performed using SAS version 9.1 (SAS Institute, Cary, NC, USA) and R version 3.0.0 (www.r-project.org). Prognostic stratification of failure-free survival (FFS; time from the first day of therapy to the day of treatment failure) by $\mathrm{T}$-classification, $\mathrm{N}$-classification and clinical stage groups was evaluated using Akaike information criterion (AIC) (Akaike, 1973) and Harrell's concordance index (c-index) (Harrell et al, 1996). The AIC was analysed using Cox proportional hazards regression model with other prognostic covariates, including age (continuous), gender, histological type, chemotherapy type and radiation technique. The optimum model - the simplest effective model with the smallest information loss when predicting outcome gives the lowest AIC value. Harrell's c-index was also calculated as a measure of predictive accuracy of survival outcome; a c-index of 0.5 indicates accuracy similar to random guessing, and that of 1.0 indicates $100 \%$ predictive accuracy. Actuarial FFS rates were estimated by the Kaplan-Meier method and survival curves were compared using the log-rank test. Multivariate analyses with covariates such as age and gender were used to calculate hazard ratios (HRs) by the Cox proportional hazards model. In addition, T-classification was included as a covariate in analyses of $\mathrm{N}$-classification, and vice versa. Two-tailed $P$-values of $<0.05$ were considered statistically significant.

\section{RESULTS}

Comparison of the TNM6th, TNM7th and TNMc2008 staging systems. Generally speaking, the most obvious differences in T-classification among the three staging systems are the distributions of nasal cavity or oropharynx involvement, paranasal sinuses extension and medial and lateral pterygoid muscle involvement. With respect to N-classification, both TNM6th and TNM7th are graded according to the palpation-based greatest diameter of 
Table 1. Classification criteria and stage groups by different systems for nasopharyngeal carcinoma

\begin{tabular}{|c|c|c|c|}
\hline The 6th edition of UICC/AJCC & The 7th edition of UICC/AJCC & $\begin{array}{l}\text { The Chinese } 2008 \text { staging } \\
\text { system }\end{array}$ & The proposed staging system \\
\hline \multicolumn{4}{|l|}{ T-classification } \\
\hline T1: nasopharynx & $\begin{array}{l}\text { T1: nasopharynx, oropharynx or } \\
\text { nasal cavity }\end{array}$ & T1: nasopharynx & T1: nasopharynx \\
\hline $\begin{array}{l}\text { T2a: oropharynx and/or nasal cavity } \\
\text { T2b: parapharyneal extension }\end{array}$ & T2: parapharyneal extension & $\begin{array}{l}\text { T2: oropharynx, nasal cavity, } \\
\text { parapharyneal extension }\end{array}$ & $\begin{array}{l}\text { T2: oropharynx, nasal cavity, } \\
\text { parapharyneal extension, medial and } \\
\text { lateral pterygoid muscles }\end{array}$ \\
\hline $\begin{array}{l}\text { T3: bony structures and/or paranasal } \\
\text { sinuses } \\
\text { T4: intracranial extension and/or } \\
\text { cranial nerves, infratemporal fossa } \\
\text { hypopharynx, orbit or masticatory } \\
\text { space }^{\text {a }}\end{array}$ & $\begin{array}{l}\text { T3: bony structures and/or } \\
\text { paranasal sinuses } \\
\text { T4: intracranial extension and/or } \\
\text { cranial nerves, hypopharynx, orbit } \\
\text { or infratemporal fossa/masticatory }_{\text {space }^{\text {b }}}\end{array}$ & $\begin{array}{l}\text { T3: skull base, medial pterygoid } \\
\text { muscle extension } \\
\text { T4: cranial nerves, paranasal sinuses, } \\
\text { masticatory space excluding medial } \\
\text { pterygoid muscle, intracranial } \\
\text { (cavernous sinus, dural meninges) } \\
\text { extension }\end{array}$ & $\begin{array}{l}\text { T3: bony structures and/or paranasal } \\
\text { sinuses } \\
\text { T4: intracranial extension and/or cranial } \\
\text { nerves, infratemporal fossa } \\
\text { hypopharynx, orbit or masticatory } \\
\text { space excluding medial and lateral } \\
\text { pterygoid muscles }\end{array}$ \\
\hline
\end{tabular}

N-classification

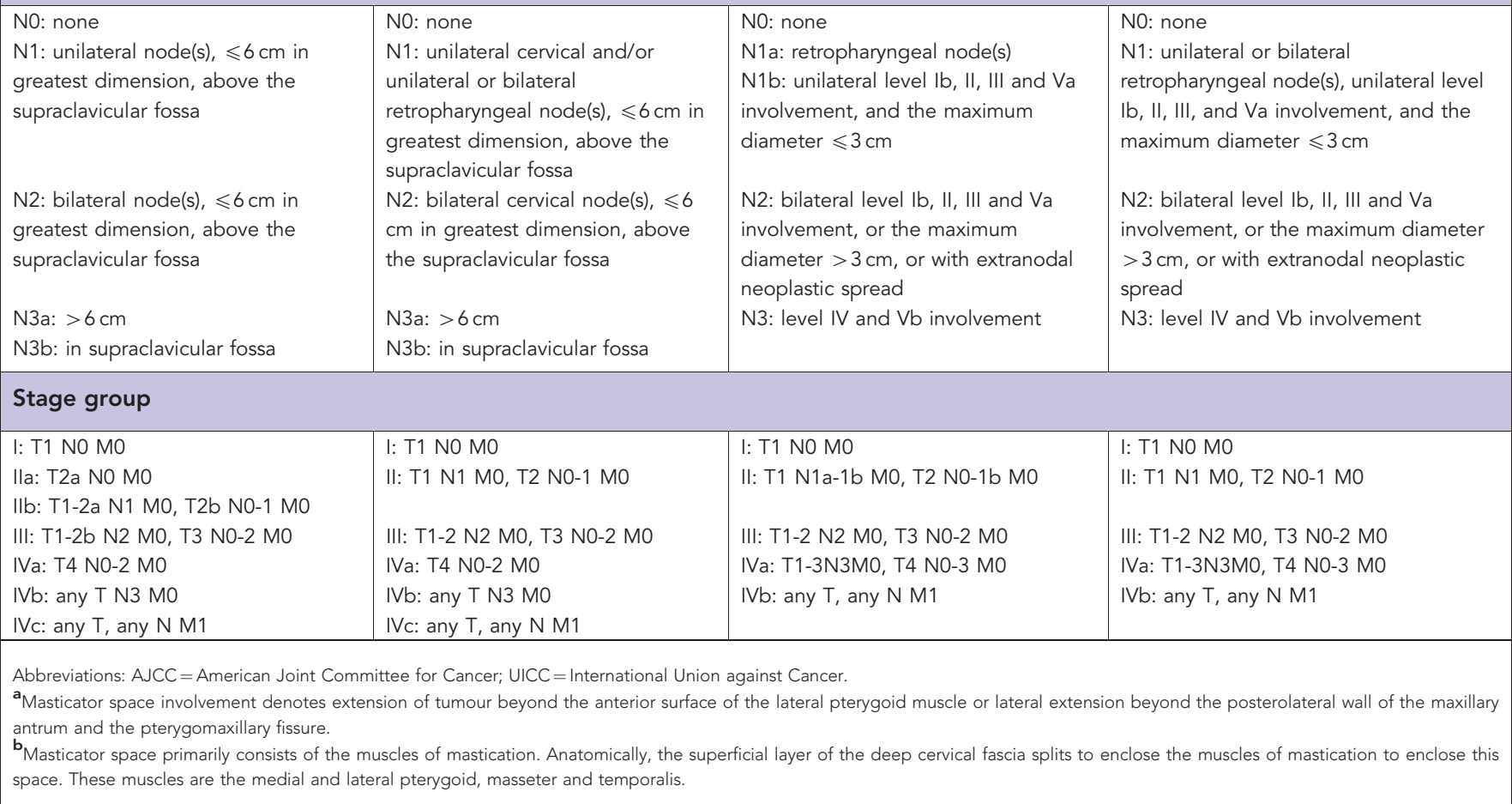

tumour-positive lymph nodes, and TNM6th disregards RP-LNs, whereas TNMc2008 is classified using MRI-determined lymph node levels and sizes, extranodal neoplastic spread (ENS) and RPLNs. In Supplementary Table 1, we have listed stage migration because of the differences among the three TNM systems for the IMRT and CRT cohorts. There were high proportions of N-classification changes $(>20 \%$ cases) between TNM6th or TNM7th and TNMc2008, and $\sim 10 \%$ of cases had T-classification changes across the three systems.

The ability of each TNM staging system to stratify FFS is presented in Table 2. In the IMRT cohort, both AIC and c-index values revealed that TNM6th had superior prognostic value by T-classification (T1/T2/T3/T4), followed by TNMc2008 or TNM7th, whereas the prognostic value by $\mathrm{N}$-classification (N0/N1/N2/N3) was highest for TNMc2008 followed by TNM7th and TNM6th. However, different trends were observed in the CRT cohort; TNMc2008 had superior prognostic value by both T-classification and N-classification, followed by TNM6th and then TNM7th. The results of the CRT cohort were validated in the third cohort. With regard to clinical stage group (I/II/III/IV), TNM6th had superior prognostic value in the IMRT cohort, followed by TNM7th and TNMc2008. In the CRT cohort, TNMc2008 was superior to TNM6th and TNM7th; this result was again validated in the third cohort.

In addition, we compared the prognostic value of the three staging systems in predicting FFS for the chemotherapy subgroups in each cohort. As shown in Table 3, in the IMRT cohort, the results of the RT alone set, CC set and IC plus CC set consistently demonstrated the highest prognostic value for T-classification of TNM6th and N-classification of TNMc2008, similar to the results obtained in the whole IMRT cohort. In the CRT cohort, the results of the RT alone set, IC set and CC set demonstrated the best prognostic value for $\mathrm{T}$-classification and $\mathrm{N}$-classification of TNMc2008, similar to the entire CRT cohort. However, in particular, in the IC plus CC set of the CRT cohort, the prognostic value of T-classification of TNM6th, rather than TNMc2008, was superior to the other staging systems, which was quite consistent with the results of the IMRT cohort. The N-classification of 
Table 2. Comparison of TNM6th, TNM7th and TNMc2008 by the proportion of patient numbers allocated in each category and the prognostic value

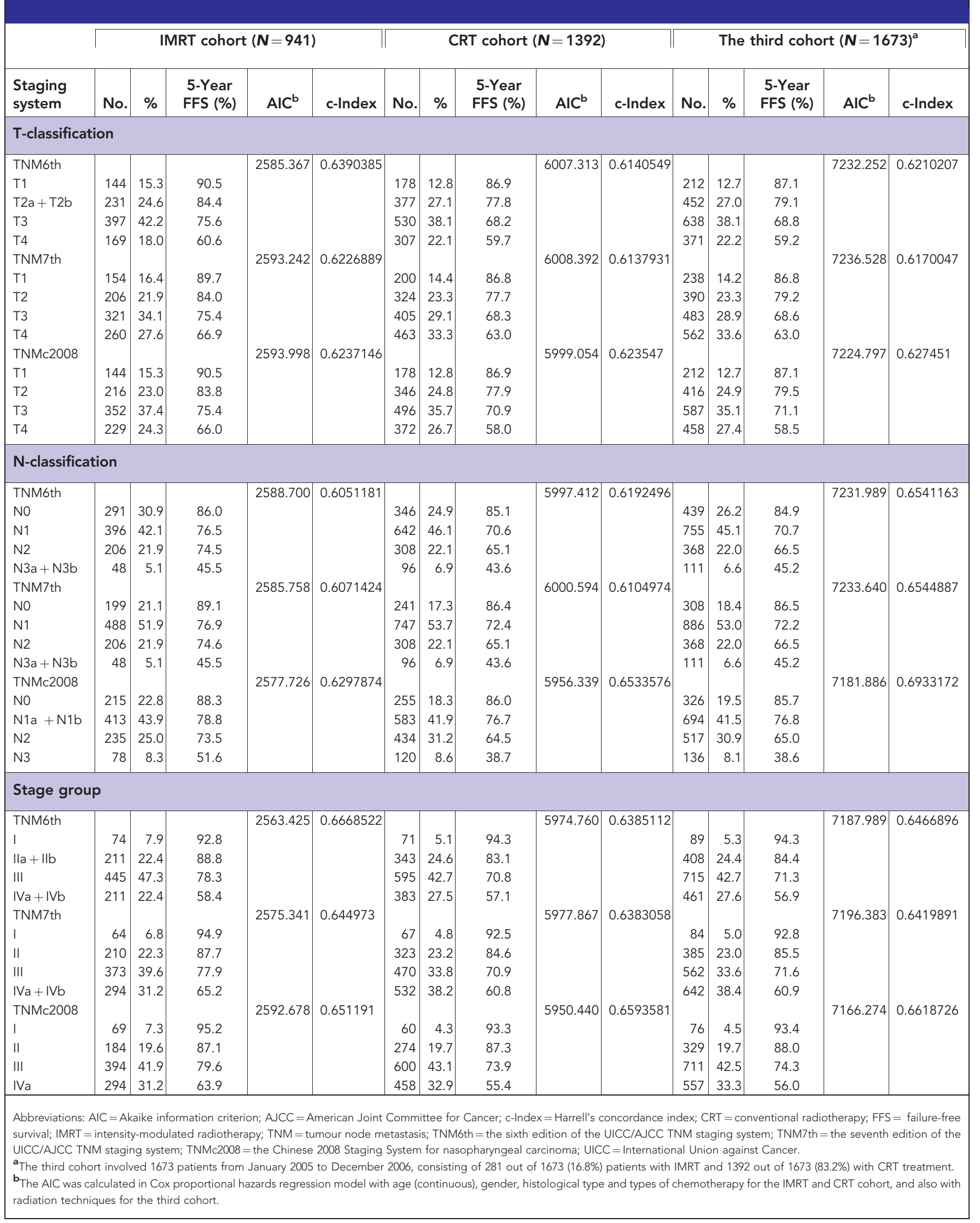


Table 3. Comparison of TNM6th, TNM7th and TNMc2008 in predicting failure-free survival in subgroups of chemotherapy

\begin{tabular}{|c|c|c|c|c|c|c|}
\hline & IMRT cohort $(N=941)$ & & CRT cohort $(N=1392)$ & & The third cohort $(N=1673)^{a}$ & \\
\hline Subgroups & $\mathrm{AIC}^{\mathrm{b}}$ & c-Index & $\mathrm{AIC}^{\mathrm{b}}$ & c-Index & $\mathrm{AIC}^{\mathrm{b}}$ & c-Index \\
\hline RT alone set & $(n=219)$ & & $(n=328)$ & & $(n=396)$ & \\
\hline \multicolumn{7}{|c|}{ T-classification } \\
\hline $\begin{array}{l}\text { TNM6th } \\
\text { TNM7th } \\
\text { TNMc2008 }\end{array}$ & $\begin{array}{l}303.375 \\
305.081 \\
304.234\end{array}$ & $\begin{array}{l}0.6878003 \\
0.6824811 \\
0.6817948\end{array}$ & $\begin{array}{l}901.218 \\
900.059 \\
896.791\end{array}$ & $\begin{array}{l}0.6621048 \\
0.6754977 \\
0.6757416\end{array}$ & $\begin{array}{l}1050.234 \\
1048.985 \\
1046.935\end{array}$ & $\begin{array}{l}0.6715685 \\
0.6806435 \\
0.6819199\end{array}$ \\
\hline
\end{tabular}

\section{N-classification}

\begin{tabular}{|l|c|c|c|c|c|c|}
\hline TNM6th & 297.935 & 0.6708991 & 901.584 & 0.6428327 & 1047.189 & 1047.472 \\
TNM7th & 294.478 & 0.6914036 & 901.148 & 0.6381977 & 1027.730 & 0.64711761 \\
TNMc2008 & 291.221 & 0.7268360 & 884.548 & 0.6748634 & 0.6870432 \\
\hline IC set & None & & $(n=281)$ & & $(n=281)$ & \\
\hline
\end{tabular}

\section{T-classification}

\begin{tabular}{|l|l|l|l|l|l|l|}
\hline TNM6th & - & - & 1083.046 & 0.5527778 & 1083.046 & 1083.147 \\
TNM7th & - & - & 1083.147 & 0.5549505 & 0.5527778 \\
TNMc2008 & - & - & 1082.190 & 0.5685369 & 1082.190 & 0.5685369 \\
\hline
\end{tabular}

$\mathrm{N}$-classification

\begin{tabular}{|l|c|c|c|c|c|c|}
\hline TNM6th & - & - & 1075.827 & 0.5958746 & 1075.827 & 1075.021 \\
TNM7th & - & - & 1075.021 & 0.5921342 & 1061.585 & 0.5921342 \\
TNMc2008 & - & - & 1061.585 & 0.6544279 & 0.6544279 \\
\hline CC set & $(n=469)$ & & $(n=423)$ & & $(n=526)$ & \\
\hline
\end{tabular}

\section{T-classification}

\begin{tabular}{|l|l|l|l|l|l|l|}
\hline TNM6th & 1187.584 & 0.5851499 & 1380.940 & 0.6128575 & 1771.414 & 1770.971 \\
TNM7th & 1189.337 & 0.5762542 & 1380.908 & 0.6148377 & 0.6162836 \\
TNMc2008 & 1191.628 & 0.5601325 & 1376.665 & 0.6290704 & 1768.39 & 0.6269293 \\
\hline
\end{tabular}

\section{N-classification}

\begin{tabular}{|l|c|l|l|l|l|l|}
\hline TNM6th & 1178.929 & 0.5946466 & 1383.465 & 0.6110286 & 0.5966301 \\
TNM7th & 1179.393 & 0.5852701 & 1383.542 & 0.5968647 & 1775.976 \\
TNMc2008 & 1174.637 & 0.6141743 & 1381.203 & 0.6186331 & 0.5876845 \\
\hline IC + CC set & $(n=186)$ & $(n=309)$ & & 0.6183843 \\
\hline
\end{tabular}

T-classification

\begin{tabular}{|c|c|c|c|c|c|c|}
\hline $\begin{array}{l}\text { TNM6th } \\
\text { TNM7th } \\
\text { TNMc2008 }\end{array}$ & $\begin{array}{l}489.122 \\
493.670 \\
491.357\end{array}$ & $\begin{array}{l}0.6492647 \\
0.6107353 \\
0.6338971\end{array}$ & $\begin{array}{l}1291.418 \\
1294.298 \\
1292.766\end{array}$ & $\begin{array}{l}0.5798540 \\
0.5564794 \\
0.5727775\end{array}$ & $\begin{array}{l}1737.786 \\
1742.804 \\
1739.083\end{array}$ & $\begin{array}{l}0.5925196 \\
0.5630775 \\
0.5865056\end{array}$ \\
\hline \multicolumn{7}{|c|}{ N-classification } \\
\hline $\begin{array}{l}\text { TNM6th } \\
\text { TNM7th } \\
\text { TNMc2008 }\end{array}$ & $\begin{array}{l}497.512 \\
497.515 \\
495.107\end{array}$ & $\begin{array}{l}0.52352941 \\
0.52294118 \\
0.55088240\end{array}$ & $\begin{array}{l}1286.780 \\
1288.125 \\
1270.375\end{array}$ & $\begin{array}{l}0.5974569 \\
0.5870632 \\
0.6623397\end{array}$ & $\begin{array}{l}1739.399 \\
1740.434 \\
1723.176\end{array}$ & $\begin{array}{l}0.5786084 \\
0.5711631 \\
0.6351195\end{array}$ \\
\hline
\end{tabular}

Abbreviations: $\mathrm{AIC}=$ Akaike information criterion; $\mathrm{AJCC}=$ American Joint Committee for Cancer; $\mathrm{CC}=$ concomitant chemotherapy; $\mathrm{c}-\mathrm{Index}=\mathrm{Harrell}$ 's concordance index; $\mathrm{CRT}=\mathrm{conventional}$ radiotherapy; IC = induction chemotherapy; IMRT = intensity-modulated radiotherapy; RT = radiotherapy; TNM=tumour node metastasis; TNM6th = the sixth edition of the UICC/AJCC TNM staging system; TNM7th $=$ the seventh edition of the UICC/AJCC TNM staging system; TNMc2008 = the Chinese 2008 Staging System for nasopharyngeal carcinoma; UICC $=$ International Union against Cancer.

a The third cohort involved 1673 patients from January 2005 to December 2006, consisting of 281 out of 1673 (16.8\%) patients with IMRT and 1392 out of 1673 (83.2\%) with CRT treatment.

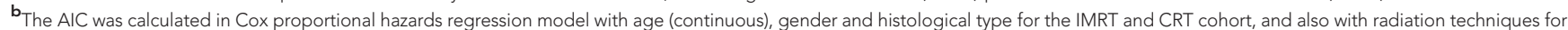
the third cohort. However, AIC and c-Index were not calculated in the RT plus CC and adjuvant chemotherapy set because of limited number of patients $(n=67$ in the IMRT cohort; $n=51$ in the CRT cohort).

TNMc2008 still had the most favourable prognostic value in the IC plus CC set of the CRT cohort. When analysed by the multiple chemotherapy subgroups, the third cohort validated the results of the CRT cohort.

In the stratums by age ( $\leqslant 60$ and $>60$ years old) and gender, the results of comparison were quite similar to those in the overall cohort, respectively (Table 4).
Comparison of staging models. Given the above differences between patients treated with IMRT and CRT, and the recent recommendation of IMRT as the standard treatment by the Head and Neck Cancers of the National Comprehensive Cancer Network (NCCN) Clinical Practice Guidelines in Oncology (Version 2, 2013; www.nccn.org), we constructed 10 T-classification models and $4 \mathrm{~N}$-classification models with the 941 patients in the IMRT 
Table 4. Comparison of TNM6th, TNM7th and TNMc2008 in predicting failure-free survival in stratified analysis by age ( $\leqslant 60$ and $>60$ years old) and gender

\begin{tabular}{l||l||l|} 
IMRT cohort $(\mathbf{N}=941)$ & CRT cohort $(\mathbf{N}=1392)$ & The third cohort $(\mathbf{N}=1673)^{\mathrm{a}}$
\end{tabular}

\begin{tabular}{|c|c|c|c|c|c|c|}
\hline Stratums & $\mathrm{AIC}^{\mathrm{b}}$ & c-Index & $A I C^{b}$ & c-Index & $A I C^{b}$ & c-Index \\
\hline Age $\leqslant 60$ years old & $(n=824)$ & & $(n=1234)$ & & $(n=1480)$ & \\
\hline \multicolumn{7}{|l|}{ T-classification } \\
\hline $\begin{array}{l}\text { TNM6th } \\
\text { TNM7th } \\
\text { TNMc2008 }\end{array}$ & $\begin{array}{l}2003.721 \\
2010.644 \\
2009.290\end{array}$ & $\begin{array}{l}0.6325691 \\
0.6175336 \\
0.6212674\end{array}$ & $\begin{array}{l}4908.478 \\
4917.419 \\
4907.862\end{array}$ & $\begin{array}{l}0.6122992 \\
0.6090601 \\
0.6194509\end{array}$ & $\begin{array}{l}5806.033 \\
5810.901 \\
5798.277\end{array}$ & $\begin{array}{l}0.6209916 \\
0.6141826 \\
0.6265544\end{array}$ \\
\hline \multicolumn{7}{|l|}{ N-classification } \\
\hline $\begin{array}{l}\text { TNM6th } \\
\text { TNM7th } \\
\text { TNMc2008 }\end{array}$ & $\begin{array}{l}2004.321 \\
2003.707 \\
1993.638\end{array}$ & $\begin{array}{l}0.6239475 \\
0.6172813 \\
0.6658321\end{array}$ & $\begin{array}{l}4901.345 \\
4903.039 \\
4854.533\end{array}$ & $\begin{array}{l}0.6239475 \\
0.6172813 \\
0.6658321\end{array}$ & $\begin{array}{l}5804.875 \\
5806.357 \\
5756.629\end{array}$ & $\begin{array}{l}0.6207027 \\
0.6149654 \\
0.658327\end{array}$ \\
\hline Age $>60$ years old & $(n=117)$ & & $(n=158)$ & & $(n=193)$ & \\
\hline
\end{tabular}

\section{T-classification}

\begin{tabular}{|l|l|l|l|l|l|l|}
\hline TNM6th & 368.177 & 0.6676618 & 712.375 & 0.6245186 & 932.624 & 0.6293492 \\
TNM7th & 369.362 & 0.6462916 & 710.596 & 0.634387 & 931.752 & 0.6357388 \\
TNMc2008 & 371.565 & 0.6296354 & 710.525 & 0.6413671 & 930.872 & 0.6368664 \\
\hline
\end{tabular}

N-classification

\begin{tabular}{|l|c|c|c|c|c|}
\hline TNM6th & 375.870 & 0.6239475 & 712.66 & 0.6046213 & 934.879 \\
TNM7th & 372.357 & 0.6090601 & 714.124 & 0.5864891 & 935.447 \\
TNMc2008 & 372.336 & 0.6658321 & 711.049 & 0.614249 & 928.596 \\
\hline Male & $(n=714)$ & & $(n=1054)$ & & $(n=1269)$ \\
\hline
\end{tabular}

T-classification

\begin{tabular}{|c|c|c|c|c|c|c|}
\hline $\begin{array}{l}\text { TNM6th } \\
\text { TNM7th } \\
\text { TNMc2008 }\end{array}$ & $\begin{array}{l}2120.131 \\
2124.745 \\
2126.928\end{array}$ & $\begin{array}{l}0.6392201 \\
0.6278203 \\
0.6222739\end{array}$ & $\begin{array}{l}4701.974 \\
4702.009 \\
4690.542\end{array}$ & $\begin{array}{l}0.6142803 \\
0.6141995 \\
0.628093\end{array}$ & $\begin{array}{l}5665.205 \\
5668.907 \\
5656.800\end{array}$ & $\begin{array}{l}0.6212674 \\
0.6175336 \\
0.6325691\end{array}$ \\
\hline \multicolumn{7}{|c|}{$\mathrm{N}$-classification } \\
\hline $\begin{array}{l}\text { TNM6th } \\
\text { TNM7th } \\
\text { TNMc2008 }\end{array}$ & $\begin{array}{l}2118.398 \\
2114.491 \\
2107.173\end{array}$ & $\begin{array}{l}0.6239475 \\
0.6172813 \\
0.6658321\end{array}$ & $\begin{array}{l}4695.677 \\
4699.371 \\
4660.241\end{array}$ & $\begin{array}{l}0.6200693 \\
0.6098774 \\
0.6546294\end{array}$ & $\begin{array}{l}5666.261 \\
5666.916 \\
5626.74\end{array}$ & $\begin{array}{l}0.6170331 \\
0.6100415 \\
0.6522923\end{array}$ \\
\hline Female & $(n=227)$ & & $(n=338)$ & & $(n=404)$ & \\
\hline \multicolumn{7}{|c|}{ T-classification } \\
\hline $\begin{array}{l}\text { TNM6th } \\
\text { TNM7th } \\
\text { TNMc2008 }\end{array}$ & $\begin{array}{l}299.740 \\
303.410 \\
300.965\end{array}$ & $\begin{array}{l}0.6435911 \\
0.6036224 \\
0.6340996\end{array}$ & $\begin{array}{l}917.401 \\
918.139 \\
919.291\end{array}$ & $\begin{array}{l}0.6128287 \\
0.6116616 \\
0.6050162\end{array}$ & $\begin{array}{l}1071.017 \\
1071.860 \\
1072.319\end{array}$ & $\begin{array}{l}0.6334405 \\
0.6270442 \\
0.6283753\end{array}$ \\
\hline \multicolumn{7}{|c|}{ N-classification } \\
\hline $\begin{array}{l}\text { TNM6th } \\
\text { TNM7th } \\
\text { TNMc2008 }\end{array}$ & $\begin{array}{l}304.453 \\
305.631 \\
304.022\end{array}$ & $\begin{array}{l}0.6239475 \\
0.6172813 \\
0.6658321\end{array}$ & $\begin{array}{l}913.893 \\
914.196 \\
907.748\end{array}$ & $\begin{array}{l}0.6180926 \\
0.6135194 \\
0.6448647\end{array}$ & $\begin{array}{l}1072.667 \\
1073.871 \\
1063.371\end{array}$ & $\begin{array}{l}0.606749 \\
0.5981399 \\
0.6396812\end{array}$ \\
\hline \multicolumn{7}{|c|}{$\begin{array}{l}\text { Abbreviations: AIC = Akaike information criterion; AJCC = American Joint Committee for Cancer; C-Index = Harrell's concordance index; CRT = conventional radiotherapy; IMRT = intensity- } \\
\text { modulated radiotherapy; TNM = tumour node metastasis; TNM6th = the sixth edition of the UICC/AJCC TNM staging system; TNM7th = the seventh edition of the UICC/AJCC TNM staging } \\
\text { system; TNMc2008 = the Chinese } 2008 \text { Staging System for nasopharyngeal carcinoma; UICC=International Union against Cancer. } \\
\text { a The third cohort involved } 1673 \text { patients from January } 2005 \text { to December 2006, consisting of } 281 \text { out of } 1673 \text { (16.8\%) patients with IMRT and } 1392 \text { out of } 1673 \text { ( } 83.2 \%) \text { with CRT treatment. } \\
\text { b The AIC was calculated in Cox proportional hazards regression model with histological type, types of chemotherapy and age (continuous) or gender for the IMRT and CRT cohort, and also } \\
\text { with radiation techniques for the third cohort. }\end{array}$} \\
\hline
\end{tabular}

cohort. The main changes of classification criteria from the based staging systems to our staging models were as follows (Supplementary Table 2). For T-classification, the first model (model-Ta1) downstaged oropharynx and/or nasal cavity involvement in TNM6th to T1, and the second (model-Ta2) downstaged oropharynx and/or nasal cavity involvement in TNMc2008 to T1. The third (model-Tb) classified oropharynx, nasal cavity and parapharyngeal extension as T1 vs TNM6th. The forth (modelTc1) and fifth (model-Tc2) categorised paranasal sinus involvement as T4 vs TNM6th, and as T3 vs TNMc2008, respectively. The sixth (model-Td1) defined lateral pterygoid muscle involvement as T4 vs TNM6th, the seventh (model-Td2) defined it as T3 vs TNM6th and the eighth (model-Td3) defined it as T3 vs TNMc2008. The ninth (model-Te1) defined medial pterygoid 
Table 5. Comparison of TNM6th and TNMc2008 staging models and the proposed staging system in predicting failure-free survival of 941 patients in the intensity-modulated radiotherapy cohort

\begin{tabular}{|c|c|c|c|c|c|c|c|}
\hline & \multicolumn{3}{|c|}{ T-classification } & \multicolumn{2}{|c|}{$\mathrm{N}$-classification } & \multicolumn{2}{|c|}{ Stage group } \\
\hline & $\begin{array}{c}\text { Based staging } \\
\text { system }\end{array}$ & $\mathrm{AIC}^{\mathrm{a}}$ & c-Index & $\mathrm{AlC}^{\mathrm{a}}$ & c-Index & $\mathrm{AIC}^{\mathrm{a}}$ & c-Index \\
\hline \multicolumn{8}{|c|}{ Staging systems } \\
\hline $\begin{array}{l}\text { TNM6th } \\
\text { TNMc2008 }\end{array}$ & & $\begin{array}{l}2585.367 \\
2593.998\end{array}$ & $\begin{array}{l}0.6390385 \\
0.6237146\end{array}$ & $\begin{array}{l}2588.700 \\
2577.726\end{array}$ & $\begin{array}{l}0.6051181 \\
0.6297874\end{array}$ & $\begin{array}{l}2563.425 \\
2592.678\end{array}$ & $\begin{array}{l}0.6668522 \\
0.6511910\end{array}$ \\
\hline \multicolumn{8}{|c|}{ Staging models } \\
\hline $\begin{array}{l}\text { Model-Ta1 } \\
\text { Model-Ta2 } \\
\text { Model-Tb } \\
\text { Model-Tc1 } \\
\text { Model-Tc2 } \\
\text { Model-Td1 } \\
\text { Model-Td2 } \\
\text { Model-Td3 } \\
\text { Model-Te1 } \\
\text { Model-Te2 } \\
\text { Model-Na } \\
\text { Model-Nb } \\
\text { Model-Nc } \\
\text { Model-Nd }\end{array}$ & $\begin{array}{l}\text { TNM6th } \\
\text { TNMc2008 } \\
\text { TNM6th } \\
\text { TNM6th } \\
\text { TNMc2008 } \\
\text { TNM6th } \\
\text { TNM6th } \\
\text { TNMc2008 } \\
\text { TNM6th } \\
\text { TNM6th } \\
\text { TNMc2008 } \\
\text { TNMc2008 } \\
\text { TNMc2008 } \\
\text { TNMc2008 }\end{array}$ & $\begin{array}{c}2585.953 \\
2594.378 \\
2590.548 \\
2588.819 \\
2592.163 \\
2590.323 \\
2585.388 \\
2589.175 \\
2590.558 \\
2587.092 \\
- \\
- \\
- \\
-\end{array}$ & $\begin{array}{c}0.6381613 \\
0.6229049 \\
0.6304656 \\
0.6344470 \\
0.6270070 \\
0.6299291 \\
0.6382018 \\
0.6320108 \\
0.6260628 \\
0.6348819 \\
- \\
- \\
-\end{array}$ & $\begin{array}{c}- \\
- \\
- \\
- \\
- \\
- \\
- \\
- \\
- \\
- \\
2582.810 \\
2600.696 \\
2601.425 \\
2601.719\end{array}$ & $\begin{array}{c}- \\
- \\
- \\
- \\
- \\
- \\
- \\
- \\
- \\
0.6203779 \\
0.6262281 \\
0.6271457 \\
0.6226484\end{array}$ & $\begin{array}{l}- \\
- \\
- \\
- \\
- \\
- \\
- \\
- \\
- \\
- \\
-\end{array}$ & $\begin{array}{l}- \\
- \\
- \\
- \\
- \\
- \\
- \\
- \\
- \\
- \\
- \\
-\end{array}$ \\
\hline $\begin{array}{l}\text { The proposed } \\
\text { staging system }\end{array}$ & & 2585.367 & 0.6390385 & 2577.726 & 0.6297874 & 2557.782 & 0.6707051 \\
\hline
\end{tabular}

muscle involvement as T4 vs TNM6th, and the last (model-Te2) defined it as T3 vs TNM6th. For N-classification, all four models were based on TNMc2008. The first (model-Na) classified positive RP-LN as stage N0, the second (model-Nb) disregarded ENS, the third (model-Nc) disregarded the size of positive cervical lymph nodes and the last (model-Nd) disregarded both ENS and cervical lymph node size.

The prognostic value of the 10 staging models for predicting FFS compared with the corresponding based TNM staging systems is presented in Table 5. For T-classification, both AIC and c-index revealed the poorer prognostic value of model-Ta1 vs TNM6th, model-Ta2 vs TNMc2008, and model-Tb vs model-Ta1; modelTc1 was inferior to TNM6th, but model-Tc2 was superior to TNMc2008. In addition, the prognostic value of model-Td1 vs TNM6th and model-Td2 vs TNM6th was lower, but model-Td3 was superior to TNMc2008. Finally, both model-Td1 and modelTd2 were inferior to TNM6th. For N-classification, all models (model-Na, model-Nb, model-Nc and model-Nd) had a poorer prognostic value than TNMc2008, and model-Nd was inferior to both model-Nb and model-Nc.

The proposed staging criteria. The above staging models failed to lead to superior stage migrations with reference to T-classification of TNM6th and N-classification of TNMc2008, and actually revalidated the superiority of these aspects of the two TNM staging systems. We therefore proposed to combine the T-classifications of the TNM6th and N-classifications of the TNMc2008 with slight modifications (merging category $\mathrm{T} 2 \mathrm{a}$ and $\mathrm{T} 2 \mathrm{~b}$ into $\mathrm{T} 2$, and category N3a and N3b into N3). (Table 1)

As indicated by the smaller AIC and larger c-index values, the clinical stage groups of the proposed staging criteria had superiority for predicting FFS in patients treated with IMRT compared with those of both TNM6th and TNMc2008 (Table 5). This observation was generally supported by the FFS curves $\left(P_{\text {trend }}<0.001\right)$ and independent significance in multivariate analysis (adjusted HR 2.14, 95\% CI 1.76-2.59, $P<0.001$ ), despite the nonsignificant increases in risk of failure between certain adjacent staging categories (stage I vs II: the log-rank test $P=0.069$; adjusted $\mathrm{HR} 3.07$, 95\% CI 0.91-10.34, $P=0.070$; Figure $1 \mathrm{C}$ and Table 6). Specifically, for T-classification and $\mathrm{N}$-classification categories, the proposed staging criteria showed to be significant predictor for FFS in univariate and multivariate analyses (T-classification: the log-rank test $P_{\text {trend }}<0.001$; adjusted HR 1.63, 95\% CI 1.38-1.92, $P<0.001$; N-classification: the logrank test $P_{\text {trend }}<0.001$; adjusted HR 1.72, 95\% CI 1.47-2.02, $P<0.001)$. When evaluating T-classification and $\mathrm{N}$-classification as categorical variables, unfortunately, we observed no significant segregations of T1 vs T2 $(P=0.063$, Figure $1 \mathrm{~A})$ and N1 vs N2 $(P=0.089$, Figure 1B).

\section{DISCUSSION}

A recent study (Pan et al, 2013) compared TNMc2008 with TNM7th in 816 Chinese patients from a single hospital. However, only 293 (35.9\%) patients underwent IMRT and there were no stratification analyses according to radiation techniques, as there was significant higher local control rate using IMRT than CRT (Lai et al, 2011). Secondly, the most recent revision of TNM7th (Edge et al, 2010; King, 2010; Sun et al, 2013) was not applied properly, as medial and lateral pterygoid muscle involvement were not classified as T4. Moreover, unfortunately, the data set was so heterogeneous - with a wide range of treatments, in particular the 

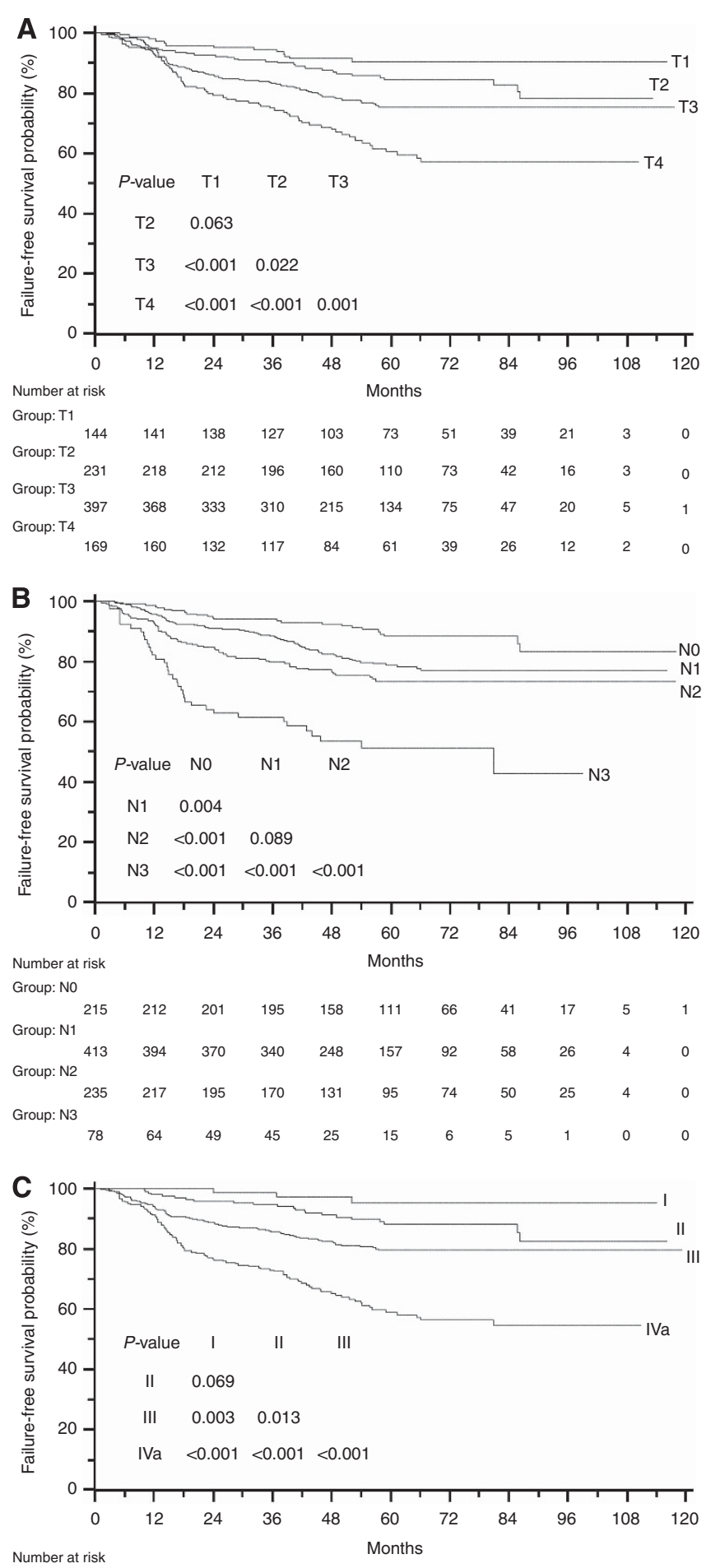

\begin{tabular}{|c|c|c|c|c|c|c|c|c|c|c|c|}
\hline Group: 1 & 69 & 69 & 69 & 67 & 57 & 39 & 24 & 19 & 11 & 2 & 0 \\
\hline \multicolumn{12}{|c|}{ Group: II } \\
\hline & 191 & 186 & 181 & 168 & 139 & 95 & 62 & 39 & 14 & 2 & 0 \\
\hline \multicolumn{12}{|c|}{ Group: III } \\
\hline & 444 & 416 & 386 & 355 & 259 & 168 & 107 & 65 & 31 & 7 & 1 \\
\hline \multicolumn{12}{|c|}{ Group: IVa } \\
\hline & 237 & 216 & 179 & 160 & 107 & 76 & 45 & 31 & 13 & 2 & 0 \\
\hline
\end{tabular}

Figure 1. Failure-free survival of 941 patients in the intensitymodulated radiotherapy cohort according to the novel proposed staging system by (A) T-classification, (B) N-classification and (C) clinical stage group.

types of chemotherapy - that credible conclusions were hard to draw without stratification analyses or Cox regression analyses. Lastly and most importantly, survival curves were delineated by
T- or N-classification of the respective staging systems, the classification categories were compared using the log-rank test and then the superiority of a staging system was judged on the basis of significant separations of the curves. In fact, survival curves are significantly affected not only by stage classification itself, but also by prognostic factors such as the treatment approach (IMRT vs CRT (Zhang et al, 2009; Lai et al, 2011; Peng et al, 2012), no chemotherapy $v s$ chemotherapy and the type of chemotherapy (Baujat et al, 2006; OuYang et al, 2013)). Therefore, it is insufficient to determine which staging system has the highest prognostic value based on survival curves in terms of $\mathrm{T}$ - or $\mathrm{N}$ - stages alone, without considering other prognoses.

In contrast, in this study we enrolled a large number of MRIbased patients $(n=2333)$, tested the staging systems in both the IMRT cohort $(n=941)$ and CRT cohort $(n=1392)$ and further validated the results in 1673 patients who were treated with either IMRT or CRT between 2005 and 2006 (Table 2). Secondly, we stratified the patients in each cohort by the major types of chemotherapy to eliminate the effects of chemotherapy heterogeneity (Table 3 ). In addition, stratified analysis on the patients' characteristics, such as age and gender, was also performed (Table 4). In essence, all staging systems are mathematical models for use in the clinic, and their ability to correctly predict patient survival must be evaluated as a whole, rather than be partially assessed based on the magnitude of segregation of each stage or category, as in previous studies (Liu et al, 2008; Mao et al, 2009; Lee et al, 2012; Chen et al, 2012b; Pan et al, 2013; Sun et al, 2013). Here, we scored the models according to their accuracy for predicting FFS in the same patients, and finally determined the superiority of the models - staging systems - according to AIC and c-index scores. In addition, patient characteristics, such as age and gender, were included in the calculation of AIC by Cox regression. Hence, our comparison of the staging systems is distinct to previous comparisons (Lee et al, 2012; Chen et al, 2012b; Pan et al, 2013; Sun et al, 2013), and more accurately and fully indicates the superiority of T-classification in TNM6th for IMRT patients, T-classification in TNMc2008 for CRT patients and $\mathrm{N}$-classification in TNMc2008 for all patients.

Considering previous controversial findings regarding the prognosis of certain structure features in TNM staging criteria, it is not unexpected to observe insignificant improvement of TNM7th over TNM6th or TNMc2008. Also, we further validated the results by staging models.

For the T-classification categories, previous studies (Lee et al, 2004; Liu et al, 2008; Mao et al, 2009) found no significant differences in locoregional relapse-free survival (LRFS) between oropharynx and/or nasal cavity involvement and tumours confined to the nasopharynx; however, the poorer prognostic values of model-Ta1 vs TNM6th and model-Ta2 vs TNMc2008 suggest these features should not be categorised as stage T1, which is supported by the report from Low et al (2004). A recent study (Lee et al, 2012) even proposed downstaging of parapharyngeal extension to $\mathrm{T} 1$, but this recommendation was not supported by comparison of model-Ta1 with model-Tb. Another study (Tang et al, 2010) concluded that anatomic masticator space involvement including the medial and lateral pterygoid muscles should be classified as stage $\mathrm{T} 4$, despite the fact that anatomic masticator space involvement was a significant independent prognostic factor for OS $(P=0.02)$, but not LRFS $(P=0.05)$ or DMFS in the same study. Our staging models (model-Td1, model-Td2, model-Td3, modelTe1 and model-Te2) also failed to improve prognostic value when pterygoid muscles involvement was classified as stage T4 or T3. In addition, there are discrepancies in categorising the paranasal sinuses between the UICC/AJCC and Chinese staging systems, but no studies had compared the prognostic value of this feature with other structures involved in T4. For the first time, model-Tc1 and model-Tc2 support the classification of paranasal sinus 
Table 6. Independent significance of T-classification, N-classification and stage group of the proposed staging system in predicting failure-free survival of 941 patients in the intensity-modulated radiotherapy cohort in multivariate analyses ${ }^{a}$

\begin{tabular}{|c|c|c|c|c|c|c|c|c|c|}
\hline & \multicolumn{3}{|c|}{ T-classification } & \multicolumn{3}{|c|}{$\mathrm{N}$-classification } & \multicolumn{3}{|c|}{ Stage-group } \\
\hline Covariates & HR & $95 \% \mathrm{Cl}$ & $\boldsymbol{P}$-value & HR & $95 \% \mathrm{Cl}$ & $\boldsymbol{P}$-value & HR & $95 \% \mathrm{Cl}$ & $P$-value \\
\hline Age (continuous) & 1.019 & $1.006-1.031$ & 0.003 & 1.020 & $1.008-1.033$ & 0.002 & 1.018 & $1.006-1.031$ & 0.003 \\
\hline Gender & 0.491 & $0.331-0.729$ & $<0.001$ & 0.490 & $0.330-0.727$ & $<0.001$ & 0.513 & $0.346-0.762$ & 0.001 \\
\hline Histological type & 1.315 & $0.551-3.142$ & 0.537 & 1.363 & $0.570-3.258$ & 0.486 & 1.567 & $0.654-3.753$ & 0.314 \\
\hline Chemotherapy & 1.015 & $0.928-1.110$ & 0.740 & 1.003 & $0.916-1.098$ & 0.943 & 1.012 & $0.923-1.109$ & 0.799 \\
\hline T-classification & & & & 1.648 & $1.389-1.956$ & $<0.001$ & & & \\
\hline $\mathrm{T} 1(n=144,15.3 \%)$ & 1.000 & & & - & - & - & - & - & - \\
\hline T2 $(n=231,24.5 \%)$ & 1.391 & $0.731-2.647$ & 0.315 & - & - & - & - & - & - \\
\hline T3 $(n=397,42.2 \%)$ & 2.245 & $1.238-4.070$ & 0.008 & - & - & - & - & - & - \\
\hline $\mathrm{T} 4(n=169,18.0 \%)$ & 3.838 & $2.076-7.094$ & $<0.001$ & - & - & - & - & - & - \\
\hline N-classification & 1.718 & $1.458-2.024$ & $<0.001$ & & & & & & \\
\hline NO $(n=215,22.8 \%)$ & - & - & - & 1.000 & & & - & - & - \\
\hline N1 $(n=413,43.9 \%)$ & - & - & - & 1.725 & $1.086-2.742$ & 0.021 & - & - & - \\
\hline $\mathrm{N} 2(n=235,25.0 \%)$ & - & - & - & 2.165 & $1.335-3.509$ & 0.002 & - & - & - \\
\hline N3 $(n=78,8.3 \%)$ & - & - & - & 6.173 & $3.627-10.507$ & $<0.001$ & - & - & - \\
\hline \multicolumn{10}{|l|}{ Stage group } \\
\hline$I(n=69,7.3 \%)$ & - & - & - & - & - & - & 1.000 & & \\
\hline II $(n=191,20.3 \%)$ & - & - & - & - & - & - & 3.074 & $0.914-10.341$ & 0.070 \\
\hline III $(n=444,47.2 \%)$ & - & - & - & - & - & - & 5.365 & $1.661-17.330$ & 0.005 \\
\hline $\operatorname{IVa}(n=237,25.2 \%)$ & - & - & - & - & - & - & 12.066 & $3.705-39.292$ & $<0.001$ \\
\hline
\end{tabular}

involvement as T3. In brief, our staging models for the T-classification category present concrete evidence of the advantages of T-classification in TNM6th without the new regrouping suggestions. The following reasons may explain the nonsignificant prognostic values of our models in contrast to previous studies (Lee et al, 2004; Liu et al, 2008; Mao et al, 2009; Tang et al, 2010; Lee et al, 2012). Firstly, there were a limited number of cases with solely oropharynx and/or nasal cavity involvement; therefore, based only on the magnitude of segregation of the survival curves by the log-rank test (Lee et al, 2004; Liu et al, 2008; Mao et al, 2009), significant differences were difficult to observe between these cases and those with tumours confined to the nasopharynx when both groups have such high survival rates. However, the slight differences between our models and TNM6th or TNMc2008 could be detected using the AIC and c-index values. Secondly, as IMRT provides better locoregional control than CRT (Zhang et al, 2009; Lai et al, 2011; Peng et al, 2012), it is reasonable that categorisation of the medial and lateral pterygoid muscles and paranasal sinuses as stage T4 did not improve prognostic values. The enhanced locoregional control offered by IMRT may also explain the phenomenon that TNM6th had the best prognostic value in the IMRT cohort, whereas TNMc2008 was superior in the CRT cohort and third cohort (Table 2). Furthermore, it is possible that the combination of IC plus CC and RT leads to an extremely high survival rate (Hui et al, 2009), which may explain why TNM6th, rather than TNMc2008, had superior prognostic value in the IC plus CC sets of the CRT cohort and third cohort (Table 3). Because of the small proportion of patients $(16.8 \%)$ treated with IMRT in the third cohort, its results were quite consistent with those of the CRT cohort.
For the N-classification categories, the MRI-based TNMc2008 was superior to the other systems as a whole, similar to the results of a previous study (Pan et al, 2013). This result was not unexpected, considering that the palpation-based greatest dimension in TNM6th and TNM7th might contain subcutaneous tissues, and the fact that differences between clinicians and their characterisation of palpated tumours can result in chaotic diversity in prognostic assessment (Lee et al, 1996; Heng et al, 1999). Because of the uncertain prognostic value of RP-LN in previous studies (Chua et al, 1997; Lu et al, 2006; Ma et al, 2007; Ng et al, 2007; Tang et al, 2008; Tham et al, 2009; Sun et al, 2013), we constructed the TNMc2008-based model-Na, and proved the importance of classifying positive RP-LN as N1. Secondly, because of the contradictory prognostic value of ENS in two studies (Mao et al, 2008; Li et al, 2013), we constructed model-Nb, and found that ENS involvement should be classified as N2. In addition, the significance of cervical lymph node size determined by clinical palpation (Lee et al, 1996; Heng et al, 1999) or MRI (Mao et al, 2008; Li et al, 2013) remained unclear; therefore, we constructed model-Nc, which demonstrated that lymph node size should not be disregarded in $\mathrm{N}$-classification. In addition, the significant roles of ENS and lymph node size were revalidated by the poorer prognostic value of model-Nd as compared with model$\mathrm{Nb}$ and model-Nc, which opposes the proposed $\mathrm{N}$-classification system by Li et al (2013). In summary, our staging models for the $\mathrm{N}$-classification categories provided concrete evidence for the advantages of $\mathrm{N}$-classification in TNMc2008 without the new regrouping suggestions.

Based on our comparison of TNM6th, TNM7th and TNMc2008, and the results of the staging models, we proposed to combine T-classification of TNM6th and N-classification of 
TNMc2008 (Table 1). The proposed staging system criteria led to a balanced clinical stage group distribution, and its clinical stage group had obviously superior prognostic value over TNM6th, TNM7th and TNMc2008 (Table 5), although no significant separation of risk of failure was observed between stage I and II $(P=0.069$, Figure 1C; $P=0.070$, Table 6).

The following limitations of this study deserve comment. Firstly, despite carefully designed step-by-step comparisons, this study was conducted on the basis of retrospective analysis of a large number of cases from a single centre; apart from 281 (16.8\%) patients from the IMRT cohort, the validation (third) cohort consisted of all the same patients in the CRT cohort, instead of the other totally different population of patients. Therefore, the results of comparing the three staging systems and especially the prognostic value of the proposed staging system need to be further confirmed in a prospective multicentre clinical study. This is what we are planning at the moment. Secondly, because the RT techniques influenced the prognostic values of T-classification, and IMRT is now recommended for NPC, our proposed staging system was constructed and validated based on cases treated with IMRT; therefore, the $\mathrm{T}$-classification and clinical stage groups of the proposed staging system may not be applicable to patients treated with CRT. Thirdly, there were nonsignificant segregations between certain adjacent stage categories using our proposed staging system. The small proportion of patients and low treatment failure rate in these stratums are important factors that should be considered. Also, this may be influenced by selection biases of patients. Further validations with data of patients from other centres are required. In addition, only the FFS was evaluated because of the large volume of data. But the events of FFS consist of locoregional relapse, distant metastasis or death, whichever is first. It is of excellence in reflecting the survival of patients, and commonly used as the primary end point in the recent randomised controlled trials. Finally, our proposed staging system had superior prognostic value over the three existing systems; unfortunately, it remains complicated. A simplified and superior staging system needs to be explored.

To summarise, TNM6th T-classification and TNMc2008 $\mathrm{N}$-classification have superior prognostic value for patients treated with IMRT; TNMc2008 T-classification and N-classification were shown to be better than TNM6th and TNM7th for patients treated with CRT. In this study, TNM7th showed no significant improvement over TNM6th. In the IMRT era, combining TNM6th T-classification and TNMc2008 N-classification with slight modifications, we propose a new staging system: (1) T-classification: T1, nasopharynx; T2, oropharynx, nasal cavity, parapharyneal extension, medial and lateral pterygoid muscles; T3, bony structures and/or paranasal sinuses; T4, intracranial extension and/or cranial nerves, infratemporal fossa hypopharynx, orbit or masticatory space excluding medial and lateral pterygoid muscles; (2) N-classification: N0, none; N1, unilateral or bilateral retropharyngeal node(s), unilateral level Ib, II, III and Va involvement and the maximum diameter $\leqslant 3 \mathrm{~cm}$; N2, bilateral level Ib, II, III and $\mathrm{Va}$ involvement or the maximum diameter $>3 \mathrm{~cm}$ or with extranodal neoplastic spread; N3, level IV, Vb involvement; (3) stage group: I, T1N0M0; II, T1N1M0, T2N0-1M0; III, T1-2N2M0, T3N0-2M0; IVa, T1-3N3M0, T4N0-3M0; and IVb, any T any N M1. Thus, TNM classification for NPC can be unified internationally and its prognostic value improved.

\section{ACKNOWLEDGEMENTS}

This work was supported by grants from the National Natural Science Foundation of China (no. 30930045) and the Science Foundation of Key Hospital Clinical Program of Ministry of Health
PR China (no. 2010-439). We greatly thank Professor Li Li and Lie Zheng (Department of Imaging Diagnosis and Interventional Center, Sun Yat-sen University Cancer Center, State Key Laboratory of Oncology in South China, Collaborative Innovation Center for Cancer Medicine) for their assistance in reviewing Magnetic Resonance Imaging.

\section{CONFLICT OF INTEREST}

The authors declare no conflict of interest.

\section{REFERENCES}

Akaike H (1973) Information Theory and an Extension of the Maximum Likelihood Principle. Akademia Kiado: Budapest, Hungary.

Baujat B, Audry H, Bourhis J, Chan AT, Onat H, Chua DT, Kwong DL, Al-Sarraf M, Chi KH, Hareyama M, Leung SF, Thephamongkhol K, Pignon JP (2006) Chemotherapy in locally advanced nasopharyngeal carcinoma: an individual patient data meta-analysis of eight randomized trials and 1753 patients. Int J Radiat Oncol Biol Phys 64(1): 47-56.

Cao SM, Simons MJ, Qian CN (2011) The prevalence and prevention of nasopharyngeal carcinoma in China. Chin J Cancer 30(2): 114-119.

CCSNPC (2013) Report on revision of the Chinese 1992 staging system for nasopharyngeal carcinoma. J Radiat Oncol 2(3): 233-240.

Chang ET, Adami HO (2006) The enigmatic epidemiology of nasopharyngeal carcinoma. Cancer Epidemiol Biomarkers Prev 15(10): 1765-1777.

Chen L, Liu L-Z, Chen M, Li W-F, Yin W-J, Lin A-H, Sun Y, Li L, Ma J (2012a) Prognostic value of subclassification using MRI in the T4 classification nasopharyngeal carcinoma intensity-modulated radiotherapy treatment. Int J Radiat Oncol Biol Phys 84(1): 196-202.

Chen L, Mao Y-P, Xie F-Y, Liu L-Z, Sun Y, Tian L, Tang L-L, Lin A-H, Li L, Ma J (2012b) The seventh edition of the UICC/AJCC staging system for nasopharyngeal carcinoma is prognostically useful for patients treated with intensity-modulated radiotherapy from an endemic area in China. Radiother Oncol 104(3): 331-337.

Chua DT, Sham JS, Kwong DL, Au GK, Choy DT (1997) Retropharyngeal lymphadenopathy in patients with nasopharyngeal carcinoma: a computed tomography-based study. Cancer 79(5): 869-877.

Edge SB, Byrd DR, Compton CC, Fritz AG, Greene FL, Trotti A (2009) AJCC Cancer Staging Manual. 7th edn (Springer): New York.

Edge SB, Byrd DR, Compton CC, Fritz AG, Greene FL, Trotti A (2010) AJCC Cancer Staging Handbook from the AJCC Cancer Staging Manual. 7th edn, Springer: New York.

Ferlay J, Bray F, Pisani P, Parkin DM (2004) Cancer Incidence, Mortality and Prevalence Worldwide. 2.0 edn, IARC Press: Lyon.

Greene FL, Page DL, Fleming ID, Fritz A, Balch CM, Haller DG, Morrow M (2002) AJCC Cancer Staging Manual. 6th edn, Chapter 4, pp 33-45. Springer: New York.

Harrell Jr FE, Lee KL, Mark DB (1996) Multivariable prognostic models: issues in developing models, evaluating assumptions and adequacy, and measuring and reducing errors. Stat Med 15: 361-387.

Heng DM, Wee J, Fong KW, Lian LG, Sethi VK, Chua ET, Yang TL, Khoo Tan HS, Lee KS, Lee KM, Tan T, Chua EJ (1999) Prognostic factors in 677 patients in Singapore with nondisseminated nasopharyngeal carcinoma. Cancer 86(10): 1912-1920.

$\mathrm{Hu}$ YC, Chang CH, Chen CH, Ger LP, Liu WS, Lin LC, Leung CM, Chang KC (2010) Impact of intracranial extension on survival in stage IV nasopharyngeal carcinoma: identification of a subset of patients with better prognosis. Jpn J Clin Oncol 41(1): 95-102.

Hui EP, Ma BB, Leung SF, King AD, Mo F, Kam MK, Yu BK, Chiu SK, Kwan WH, Ho R, Chan I, Ahuja AT, Zee BC, Chan AT (2009) Randomized phase II trial of concurrent cisplatin-radiotherapy with or without neoadjuvant docetaxel and cisplatin in advanced nasopharyngeal carcinoma. J Clin Oncol 27(2): 242-249.

King AD (2010) Magnetic resonance imaging staging of nasopharyngeal carcinoma in the head and neck. World J Radiol 2(5): 159.

Lai SZ, Li WF, Chen L, Luo W, Chen YY, Liu LZ, Sun Y, Lin AH, Liu MZ, Ma J (2011) How does intensity-modulated radiotherapy versus conventional two-dimensional radiotherapy influence the treatment results in 
nasopharyngeal carcinoma patients? Int J Radiat Oncol Biol Phys 80(3): 661-668.

Lee AW, Au JS, Teo PM, Leung TW, Chua DT, Sze WM, Zee BC, Law SC, Leung SF, Tung SY, Kwong DL, Lau WH (2004) Staging of nasopharyngeal carcinoma: suggestions for improving the current UICC/AJCC Staging System. Clin Oncol (R Coll Radiol) 16(4): 269-276.

Lee AW, Foo W, Poon YF, Law CK, Chan DK, O SK, Tung SY, Ho JH (1996) Staging of nasopharyngeal carcinoma: evaluation of $\mathrm{N}$-staging by $\mathrm{Ho}$ and UICC/AJCC systems. Union Internationale Contre le Cancer. American Joint Committee for Cancer. Clin Oncol (R Coll Radiol) 8(3): 146-154.

Lee AWM, Ng WT, Chan LK, Chan OSH, Hung WM, Chan CC, Cheng PTC, Sze H, Lam TS, Yau TK (2012) The strength/weakness of the AJCC/UICC staging system (7th edition) for nasopharyngeal cancer and suggestions for future improvement. Oral Oncol 48(10): 1007-1013.

Li WF, Sun Y, Mao YP, Chen L, Chen YY, Chen M, Liu LZ, Lin AH, Li L, Ma J (2013) Proposed lymph node staging system using the international consensus guidelines for lymph node levels is predictive for nasopharyngeal carcinoma patients from endemic areas treated with intensity modulated radiation therapy. Int J Radiat Oncol Biol Phys 86(2): 249-256.

Liang SB, Sun Y, Liu LZ, Chen Y, Chen L, Mao YP, Tang LL, Tian L, Lin AH, Liu MZ, Li L, Ma J (2009) Extension of local disease in nasopharyngeal carcinoma detected by magnetic resonance imaging: improvement of clinical target volume delineation. Int J Radiat Oncol Biol Phys 75(3): $742-750$.

Liu M-Z, Tang L-L, Zong J-F, Huang Y, Sun Y, Mao Y-P, Liu L-Z, Lin A-H, Ma J (2008) Evaluation of sixth edition of AJCC staging system for nasopharyngeal carcinoma and proposed improvement. Int J Radiat Oncol Biol Phys 70(4): 1115-1123.

Low JS, Heng DM, Wee JT (2004) The question of T2a and N3a in the UICC/AJCC (1997) staging system for nasopharyngeal carcinoma. Clin Oncol (R Coll Radiol) 16(8): 581-583.

Lu JC, Wei BQ, Chen WZ, Qian PD, Zhang YQ, Wei Q, Cha WW, Li F, Ni M (2006) Staging of nasopharyngeal carcinoma investigated by magnetic resonance imaging. Radiother Oncol 79(1): 21-26.

Ma J, Liu L, Tang L, Zong J, Lin A, Lu T, Cui N, Cui C, Li L (2007) Retropharyngeal lymph node metastasis in nasopharyngeal carcinoma: prognostic value and staging categories. Clin Cancer Res 13(5): $1445-1452$.

Mao Y-P, Xie F-Y, Liu L-Z, Sun Y, Li L, Tang L-L, Liao X-B, Xu H-Y, Chen L, Lai S-Z, Lin A-H, Liu M-Z, Ma J (2009) Re-evaluation of 6th edition of AJCC staging system for nasopharyngeal carcinoma and proposed improvement based on magnetic resonance imaging. Int J Radiat Oncol Biol Phys 73(5): 1326-1334.

Mao YP, Liang SB, Liu LZ, Chen Y, Sun Y, Tang LL, Tian L, Lin AH, Liu MZ, Li L, Ma J (2008) The N staging system in nasopharyngeal carcinoma with radiation therapy oncology group guidelines for lymph node levels based on magnetic resonance imaging. Clin Cancer Res 14(22): 7497-7503.

Ng WT, Lee AW, Kan WK, Chan J, Pang ES, Yau TK, Lau KY (2007) $\mathrm{N}$-staging by magnetic resonance imaging for patients with nasopharyngeal carcinoma: pattern of nodal involvement by radiological levels. Radiother Oncol 82(1): 70-75.

OuYang PY, Xie C, Mao YP, Zhang Y, Liang XX, Su Z, Liu Q, Xie FY (2013) Significant efficacies of neoadjuvant and adjuvant chemotherapy for nasopharyngeal carcinoma by meta-analysis of published literature-based randomized, controlled trials. Ann Oncol 24(8): 2136-2146.

Pan J, Xu Y, Qiu S, Zong J, Guo Q, Zhang Y, Lin S, Lu JJ (2013) A comparison between the Chinese 2008 and the 7th edition AJCC staging systems for nasopharyngeal carcinoma. Am J Clin Oncol; e-pub ahead of print 19 April 2013; doi:10.1097/COC.0b013e31828f5c96.

Peng G, Wang T, Yang KY, Zhang S, Zhang T, Li Q, Han J, Wu G (2012) A prospective, randomized study comparing outcomes and toxicities of intensity-modulated radiotherapy vs. conventional two-dimensional radiotherapy for the treatment of nasopharyngeal carcinoma. Radiother Oncol 104(3): 286-293.

Sun R, Qiu HZ, Mai HQ, Zhang Q, Hong MH, Li YX, Yang J, Sun J, Mo HY (2013) Prognostic value and differences of the sixth and seventh editions of the UICC/AJCC staging systems in nasopharyngeal carcinoma. J Cancer Res Clin Oncol 139(2): 307-314.

Tang L, Li L, Mao Y, Liu L, Liang S, Chen Y, Sun Y, Liao X, Tian L, Lin A, Liu M, Ma J (2008) Retropharyngeal lymph node metastasis in nasopharyngeal carcinoma detected by magnetic resonance imaging: prognostic value and staging categories. Cancer 113(2): 347-354.

Tang LL, Li WF, Chen L, Sun Y, Chen Y, Liu LZ, Mao YP, Lin AH, Li L, Ma J (2010) Prognostic value and staging categories of anatomic masticator space involvement in nasopharyngeal carcinoma: a study of 924 cases with MR imaging. Radiology 257(1): 151-157.

Tham IW-K, Hee SW, Yap SP, Tuan JK-L, Wee J (2009) Retropharyngeal nodal metastasis related to higher rate of distant metastasis in patients with N0 and N1 nasopharyngeal cancer. Head Neck 31(4): 468-474.

Wei WI, Sham JS (2005) Nasopharyngeal carcinoma. Lancet 365(9476): 2041-2054.

Zhang Y, Lin ZA, Pan JJ, Zheng Z, Yang L, Lin SJ, Zheng F (2009) [Concurrent control study of different radiotherapy for primary nasopharyngeal carcinoma: intensity-modulated radiotherapy versus conventional radiotherapy]. Ai Zheng 28(11): 1143-1148.

This work is published under the standard license to publish agreement. After 12 months the work will become freely available and the license terms will switch to a Creative Commons AttributionNonCommercial-Share Alike 3.0 Unported License.

Supplementary Information accompanies this paper on British Journal of Cancer website (http://www.nature.com/bjc) 